\title{
ON THE DIFFERENTIAL EQUATION TO A CURVE OF ANY ORDER.
}

[Nature, xxxiv. (1886), pp. 365, 366.]

To Mr Samuel Roberts (see Reprint of Educational Times, x. p. 47) is due the credit of having been the first to show that a direct method of elimination properly conducted leads to the differential equation for a cubic curve; but he has not attempted to obtain the general formula for a curve of any order. By aid of a very simple idea explained in a paper intended to appear in the Comptes Rendus of the Institute, I find * without calculation the general form of this equation. The left-hand member of it may be conveniently termea the differential criterion to the curve. One single matrix will then serve to express the criteria for all curves'whose order does not exceed any prescribed number. For instance, suppose we wish to have the criteria for the orders $1,2,3,4:-$

Let $m \cdot \mu$ be used in general to denote the coefficient of $h^{m}$ in

$$
\left(\frac{1}{1.2} y^{\prime \prime} h^{2}+\frac{1}{1.2 .3} y^{\prime \prime \prime} h^{3}+\frac{1}{1.2 .3 .4} y^{\prime \prime \prime \prime} h^{4}+\ldots\right)^{\mu} .
$$

Write down the matrix-

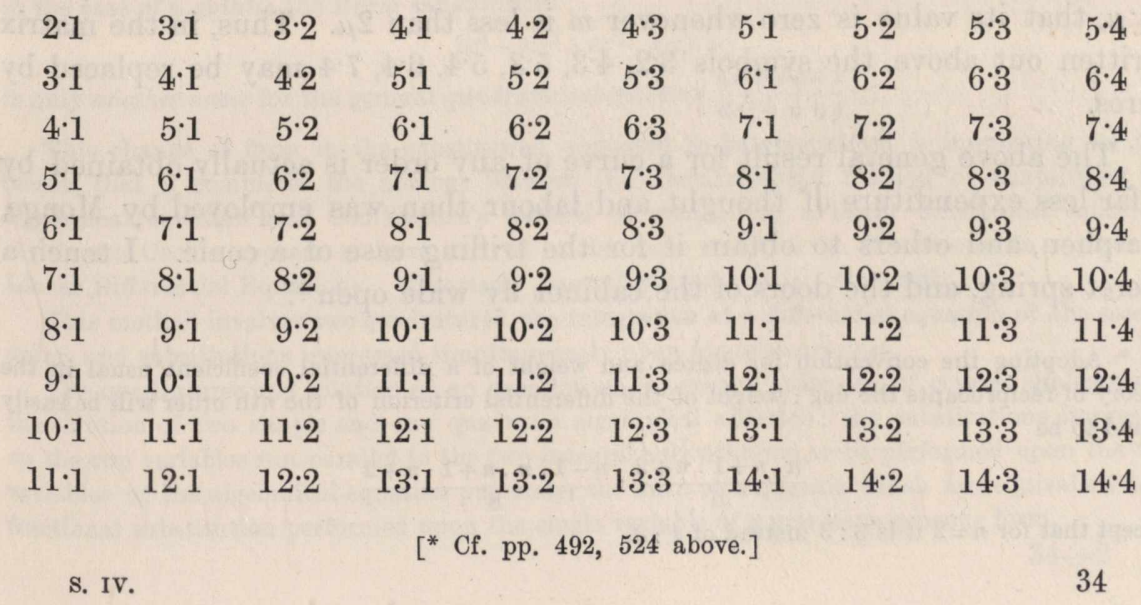


The determinant of the entire matrix, which is of the tenth order, is the criterion for a quartic curve. The determinant of the minor of the sixth order, comprised within the first six lines and columns, is the criterion for a cubic. The determinant of the third order, comprised within the first three lines and columns (subject to a remark about to be made) will furnish the criterion for a conic, and the apex of the matrix is the criterion for the straight line. By adding on five more lines and columns, according to an obvious law, the matrix may be extended so as to give the criterion for a quintic; then six more lines and columns a sextic, and so on as far as may be required.

The remark to be made concerning the determinant of the third order referred to is that it contains the irrelevant factor $2 \cdot 1$, that is, $\frac{y^{\prime \prime}}{2}$, so that the criterion for a conic (Monge's) is this determinant divested of such factor. It is certain that the next determinant is indecomposable, and is therefore the criterion for a cubic. There is no reason that I know of to suppose that any other determinant except that one which corresponds to the conic, is decomposable into factors. If this is made out, then, observing that the single term which is the criterion for the right line is indecomposable, we have another example of what may be called, in Babbage's words, a miraculous exception to a general law.

A well-known similar case of such miraculous exception I had occasion many years ago to notice in connection with the criteria for determining the number of real and imaginary roots in an algebraical equation. Such criteria may, with one single exception, be expressed by means of invariants. The case of exception is the biquadratic equation, for which it is impossible to assign an invariantive criterion that shall serve to distinguish between the cases of all the roots being real and all imaginary.

It is proper to notice that it follows, from the definition of the symbol $m \cdot \mu$, that its value is zero whenever $m$ is less than $2 \mu$. Thus, in the matrix written out above, the symbols $3 \cdot 2,4 \cdot 3,5 \cdot 3,5 \cdot 4,6 \cdot 4,7 \cdot 4$ may be replaced by zeros.

The above general result for a curve of any order is actually obtained by a far less expenditure of thought and labour than was employed by Monge, Halphen, and others to obtain it for the trifling case of a conic. I touch a secret spring, and the doors of the cabinet fly wide open*.

* Adopting the convention for degree and weight of a differential coefficient usual in the theory of reciprocants the deg : weight of the differential criterion of the $n$th order will be easily found to be

$$
\frac{n \cdot n+1 \cdot n+2}{6}: \frac{n-1 \cdot n \cdot n+1 \cdot n+2}{8}
$$

except that for $n=2$ it is $3: 3$ instead of $4: 3$. 